\title{
THE RADII OF STARLIKENESS AND CONVEXITY OF ORDER ALPHA OF TYPICALLY REAL FUNCTIONS
}

\author{
PAVEL G. TODOROV \\ Dedicated To My Parents
}

Let $T R$ denote the class of typically real functions

(1)

$$
f(z)=\int_{-1}^{1} \frac{z}{1-2 t z+z^{2}} d \mu(t)
$$

in the unit disc $|z|<1$, where $\mu(t)$ is a probability measure on $[-1,1]$. Kirwan [1] has found that the radius of starlikeness of the class $T R$ is the number $\sqrt{2}-1$, i.e. for each function $f \in T R$ the inequality

$$
\operatorname{Re} \frac{z f^{\prime}(z)}{f(z)} \geqq 0
$$

holds in the disc $|z| \leqq \sqrt{2}-1$, with equality only for the function

$$
f(z)=\frac{z\left(1+z^{2}\right)}{\left(1-z^{2}\right)^{2}}=\sum_{n=1}^{\infty}(2 n-1) z^{2 n-1}
$$

at the points $z= \pm i(\sqrt{2}-1)$.

In this paper we shall find the radii of starlikeness and convexity of an arbitrary order $\alpha, 0 \leqq \alpha<1$, of the class $T R$. In particular, for $\alpha=0$ we obtain again the Kirwan result by a new method (cf. [1] and [3]) and the heretofore unknown radius of convexity of the class $T R$ reported in [4].

We denote by $r_{s}(\alpha), 0 \leqq \alpha<1$, the radius of starlikeness of order $\alpha$ in $T R$, i.e. $r_{s}(\alpha)$ is the maximum of the numbers $r$ such that the inequality

$$
\operatorname{Re} \frac{z f^{\prime}(z)}{f(z)} \geqq \alpha
$$

holds for each function $f \in T R$ in $|z| \leqq r$. 
Theorem 1. In the class TR of typically real functions in the unit disc the radius of starlikeness of order $\alpha$ is obtained from the formulae

$$
\begin{gathered}
r_{s}(\alpha)=\sqrt{\frac{\sqrt{\alpha^{2}+8}-\alpha-2}{\sqrt{\alpha^{2}+8}-\alpha+2}} \quad \text { if } 0 \leqq \alpha<1 / \sqrt{3} \\
r_{s}(1 / \sqrt{3})=2-\sqrt{3} \quad \text { if } \quad \alpha=1 / \sqrt{3} \\
r_{s}(\alpha)=\frac{1-\alpha}{1+\alpha} \quad \text { if } \quad 1 / \sqrt{3}<\alpha<1 .
\end{gathered}
$$

Equality holds only for the following extremal functions:

1) in the case $0 \leqq \alpha<1 / \sqrt{3}$ for the function (2) at the "critical points" $z= \pm i r_{s}(\alpha)$.

2) in the case $\alpha=1 / \sqrt{3}$ for the function (2) at the "critical points" $z= \pm i r_{s}(1 / \sqrt{3})$ and for the Koebe functions

$$
f(z)=\frac{z}{(1 \pm z)^{2}}=\sum_{n=1}^{\infty}(\mp 1)^{n-1} n z^{n}
$$

at the "critical points" $z= \pm r_{s}(1 \sqrt{3})$, respectively.

3 ) in the case $1 / \sqrt{3}<\alpha<1$ for the Koebe functions (6) at the "critical points" $z= \pm r_{s}(\alpha)$, respectively.

Proof. By a theorem of Ruscheweyh ([2], Theorem 1a, pp. 19-20), the minimum of the functional $\operatorname{Re}\left[z f^{\prime}(z) / f(z)\right]$ in the class $T R$ at a fixed point $z$ is attained for functions of the form

$$
f(z)=\frac{\mu z}{1-2 t_{1} z+z^{2}}+\frac{(1-\mu) z}{1-2 t_{2} z+z^{2}} \in T R, \quad 0 \leqq \mu \leqq 1, \quad-1 \leqq t_{1} \leqq t_{2} \leqq 1 .
$$

From (7) we obtain

$$
f(z)=\frac{z\left(1-2 \tau_{s} z+z^{2}\right)}{\left(1-2 t_{1} z+z^{2}\right)\left(1-2 t_{2} z+z^{2}\right)}, \quad \tau_{s}=(1-\mu) t_{1}+\mu t_{2} .
$$

Hence

$$
\frac{z f^{\prime}(z)}{f(z)}=\gamma\left(t_{1}, z\right)+\gamma\left(t_{2}, z\right)-\gamma\left(\tau_{s}, z\right)
$$

where

$$
\gamma(u, z):=\frac{1-z^{2}}{1-2 u z+z^{2}}
$$

From (9) it follows that

where

$$
\operatorname{Re} \frac{z f^{\prime}(z)}{f(z)}=F\left(t_{1}, z\right)+F\left(t_{2}, z\right)-F\left(\tau_{s}, z\right)
$$

$$
F(u, z):=\operatorname{Re} \gamma(u, z)
$$


For a fixed $z \neq 0,|z|<1$ we write

$$
m_{s}(z)=\min _{I_{s}}\left(F\left(t_{1}, z\right)+F\left(t_{2}, z\right)-F\left(\tau_{s}, z\right)\right)
$$

where

$$
I_{s}=\left\{\left(t_{1}, \tau_{s}, t_{2}\right) \mid-1 \leqq t_{1} \leqq \tau_{s} \leqq t_{2} \leqq 1\right\} .
$$

From (10) and (11) we obtain for $z=r e^{i \theta}, 0<r<1,-\pi \leqq \theta \leqq \pi$ and real $u=t,-1 \leqq t \leqq 1$

where

$$
F(t, z)=\frac{\varrho(\sigma-t \cos \theta)}{t^{2}-2 \sigma t \cos \theta+\sigma^{2}-\sin ^{2} \theta}
$$

$$
\sigma:=\operatorname{ch}\left(\ln \frac{1}{r}\right)=\frac{1}{2}\left(\frac{1}{r}+r\right), \quad \varrho:=\operatorname{sh}\left(\ln \frac{1}{r}\right)=\frac{1}{2}\left(\frac{1}{r}-r\right) .
$$

By investigating the sign of the derivative $(d / d t) F(t, z)$, depending on $t$, we conclude that for a fixed $z \neq 0,|z|<1$, on the interval $-1 \leqq t \leqq 1$ the function $F(t, z)$ has the following properties:

a) It is strictly increasing if $0 \leqq|\theta| \leqq \psi(\sigma)$, where

$$
\cos \psi(\sigma)=\frac{\sqrt{\sigma^{2}+8}-\sigma}{2}, \quad 0<\psi(\sigma)<\frac{\pi}{2} \quad(\sigma>1)
$$

b) it is strictly decreasing if $\pi-\psi(\sigma) \leqq|\theta| \leqq \pi$, and

c) it is strictly increasing on $\left[-1, t_{0}\right]$ and strictly decreasing on $\left[t_{0}, 1\right]$ if $\psi(\sigma)<|\theta|<\pi-\psi(\sigma)$, where

$$
t_{0}=\frac{\cos \theta\left(\sigma^{2}+\sin ^{2} \theta\right)}{\sigma+|\sin \theta| \sqrt{\sigma^{2}-\cos ^{2} \theta}} \quad\left(-1<t_{0} \div 1\right)
$$

and

$$
F\left(t_{0}, z\right)=\frac{\sigma}{2 \varrho}+\frac{\sqrt{\sigma^{2}-\cos ^{2} \theta}}{2 \varrho|\sin \theta|} .
$$

Thus, from a), b), c), (15-17), (12-13) and (7--8) we can draw the following conclusions.

(i) If $0<r<1$ and $0 \leqq|\theta| \leqq \psi(\sigma)$, then

$$
m_{s}(z)=\min _{I_{s}}\left(F\left(t_{1}, z\right)+\left(F\left(t_{2}, z\right)-F\left(\tau_{s}, z\right)\right)\right)=F(-1, z)=\frac{\varrho}{\sigma+\cos \theta}
$$

with the extremal function $f(z)=z /(1+z)^{2}$. From (18) it follows that

$$
m_{s}\left(r e^{i \theta}\right) \geqq m_{s}(r)=\frac{\varrho}{\sigma+1}, \quad 0<r<1, \quad 0 \leqq|\theta| \leqq \psi(\sigma),
$$

where the equality holds only for $\theta=0$.

(ii) If $0<r<1$ and $\pi-\psi(\sigma) \leqq|\theta| \leqq \pi$, then

$$
m_{s}(z)=\min _{I_{s}}\left(\left(F\left(t_{1}, z\right)-F\left(\tau_{s}, z\right)\right)+F\left(t_{2}, z\right)\right)=F(1, z)=\frac{\varrho}{\sigma-\cos \theta}
$$


with the extremal function $f(z)=z /(1-z)^{2}$. From (20) it follows that

$$
m_{s}\left(r e^{i \theta}\right) \geqq m_{s}(-r)=\frac{\varrho}{\sigma+1}, \quad 0<r<1, \quad \pi-\psi(\sigma) \leqq|\theta| \leqq \pi,
$$

where the equality holds only for $|\theta|=\pi$.

(iii) If $0<r<1$ and $\psi(\sigma)<|\theta|<\pi-\psi(\sigma)$ and if we choose in (13)

$$
\begin{gathered}
t_{1}=-1, \quad \tau_{s}=t_{0}, \quad t_{2}=1, \\
m_{s}(z)=F(-1, z)+F(1, z)-F\left(t_{0}, z\right) \\
=\frac{2 \sigma \varrho}{\sigma^{2}-\cos ^{2} \theta}-\frac{\sigma}{2 \varrho}-\frac{\sqrt{\sigma^{2}-\cos ^{2} \theta}}{2 \varrho|\sin \theta|}=: g(\theta),
\end{gathered}
$$

then

where the extremal function is determined by (8) with (22).

For $0<\theta \leqq \pi / 2$ the function on the right-hand side of (23) has a maximum if $1<\sigma<2 \sqrt{2}$, and it increases if $\sigma \geqq 2 \sqrt{2}$. The maximum is obtained on the interval $\psi(\sigma)<\theta<\pi / 2$. Hence for $\psi(\sigma) \leqq \theta \leqq \pi / 2$ we have

$$
g(\theta) \geqq \min \left(g(\psi(\sigma)), g\left(\frac{\pi}{2}\right)\right), \quad 1<\sigma<2 \sqrt{2},
$$

and

$$
g(\theta) \geqq g(\psi(\sigma)), \quad \sigma \geqq 2 \sqrt{2},
$$

where

$$
g(\psi(\sigma))=\frac{\varrho\left(\sqrt{\sigma^{2}+8}-\sigma\right)}{4}, \quad \sigma>1,
$$

and

$$
g\left(\frac{\pi}{2}\right)=\frac{2 \varrho}{\sigma}-\frac{\sigma}{\varrho}, \quad 1<\sigma<2 \sqrt{ } 2 .
$$

Thus for $\psi(\sigma)<|\theta|<\pi-\psi(\sigma)$ we obtain from (23-27) and (14-15)

$$
m_{s}\left(r e^{i \theta}\right) \geqq m_{s}( \pm i r)=\frac{2 \varrho}{\sigma}-\frac{\sigma}{\varrho}, \quad 1<\sigma \leqq \sqrt{\frac{7+\sqrt{17}}{2}} .
$$

where the equality holds only for $|\theta|=\pi / 2$, and

$$
m_{s}\left(r e^{i \theta}\right)>\frac{\varrho\left(\sqrt{\sigma^{2}+8}-\sigma\right)}{4}, \quad \sigma>\sqrt{\frac{7+\sqrt{17}}{2}} .
$$

Now we compare the "minima" in (19), (21), (28) and (29). On the circle $|z|=r$ we have

$$
m_{s}(z) \geqq \frac{2 \varrho}{\sigma}-\frac{\sigma}{\varrho}, \quad 1<\sigma<2 \quad(2-\sqrt{3}<r<1),
$$


where the equality holds only for $z= \pm i r$,

$$
m_{s}(z) \geqq \frac{1}{\sqrt{3}}, \quad \sigma=2(r=2-\sqrt{3}),
$$

where the equality holds only for $z= \pm i(2-\sqrt{3})$ and $z=t(2-\sqrt{3})$, and

$$
m_{s}(z) \geqq \frac{\varrho}{\sigma+1}, \quad \sigma>2 \quad(0<r<2-\sqrt{3}),
$$

where the equality holds only for $z= \pm r$, respectively.

Finally, if we equate with $\alpha(0 \leqq \alpha<1)$ the "minima" $(30-32)$ for $\sigma \geqq \sqrt{2}$, we obtain the radii (3-5) of the discs in which the inequalities $\operatorname{Re}\left(z f^{\prime}(z) / f(z)\right) \geqq \alpha$, $0 \leqq \alpha<1$, hold with the extremal functions (2) and (6), respectively.

This completes the proof of Theorem 1.

In particular, for $\alpha=0$ we obtain from our general formula (3) the well-known radius of starlikeness $r_{s}:=r_{s}(0)=\sqrt{2}-1$ of the class (1) found by Kirwan [1] in a way, which is, in our opinion, difficult. See also [3] for a simple proof of this Kirwan theorem by an alternate method.

From (30-32) and (14) we obtain also the following sharp estimates for $\operatorname{Re}\left(z f^{\prime}(z) / f(z)\right)$ in the class $T R$ :

Theorem 2. For each typically real function (1) in the ring $2-\sqrt{3}<r=|z|<1$ the inequality

$$
\operatorname{Re} \frac{z f^{\prime}(z)}{f(z)} \geqq \frac{1-6 r^{2}+r^{4}}{1-r^{4}}
$$

holds, with equality only for the function (2) at the points $z= \pm i r$. On the circle $|z|=2-\sqrt{3}$

$$
\operatorname{Re} \frac{z f^{\prime}(z)}{f(z)} \geqq \frac{1}{\sqrt{3}},
$$

with equality only for the function (2) at the points $z= \pm i(2-\sqrt{3})$ and for the functions (6) at the points $z= \pm(2-\sqrt{3})$. In the disc $0 \leqq r=|z|<2-\sqrt{3}$ the inequality

$$
\operatorname{Re} \frac{z f^{\prime}(z)}{f(z)} \geqq \frac{1-r}{1+r}
$$

holds, where for $r \neq 0$ the equality holds only for the functions (6) at the points $z= \pm r$.

Theorem 2 yields the following sharp estimates in the class $T R$ :

Theorem 3. For each typically real function (1), we have under the conditions $2-\sqrt{3} \leqq \varrho \leqq r \leqq \sqrt{2}-1$, and $-\pi \leqq \theta \leqq \pi$

$$
\left|\frac{f\left(r e^{i \theta}\right)}{f\left(\varrho e^{i \theta}\right)}\right| \geqq \frac{r\left(1-r^{2}\right)}{\left(1+r^{2}\right)^{2}} \cdot \frac{\left(1+\varrho^{2}\right)^{2}}{\varrho\left(1-\varrho^{2}\right)},
$$


where for $r \neq \varrho$ the equality holds only for the function (2) with $|\theta|=\pi / 2$. Under the conditions $0<\varrho \leqq r \leqq 2-\sqrt{3} \mid$ and $-\pi \leqq \theta \leqq \pi$,

$$
\left|\frac{f\left(r e^{i \theta}\right)}{f\left(\varrho e^{i \theta}\right)}\right| \geqq \frac{r}{(1+r)^{2}} \cdot \frac{(1+\varrho)^{2}}{\varrho},
$$

with equality for $r \neq \varrho$ only for the functions (6) for $\theta=0$ and $|\theta|=\pi$, respectively.

Proof. The theorem follows if we use the identity

$$
\operatorname{Re} \frac{z f^{\prime}(z)}{f(z)}=r \frac{\partial}{\partial r} \ln |f(z)|,
$$

and integrate the inequalities (33-35) with respect to $r$ from $\varrho \geqq 2-\sqrt{3}$ to $r \leqq \sqrt{2}-1$ and from $\varrho>0$ to $r \leqq 2-\sqrt{3}$, respectively.

Now we shall find in $T R$ the radius of convexity of order $\alpha, 0 \leqq \alpha<1$, which we denote by $r_{c}(\alpha)$. By definition, $r_{c}(\alpha)$ is the maximum of the numbers $r$ such that

$$
\operatorname{Re}\left(1+\frac{z f^{\prime \prime}(z)}{f^{\prime}(z)}\right) \geqq \alpha
$$

for every function $f \in T R$ in $|z| \leqq r$.

Theorem 4. Let $\alpha_{0}=0.314 \ldots$ be the least positive root of the equation

$$
x^{6}-17 x^{4}-120 x^{2}+12=0 .
$$

Then for $0 \leqq \alpha \leqq \alpha_{0}$, the radius $r_{c}(\alpha)$ is equal to the least positive root of the equation

$$
(1+\alpha) r^{6}-(23+5 \alpha) r^{4}+(23-5 \alpha) r^{2}-(1-\alpha)=0 .
$$

In particular, for $\alpha=\alpha_{0}$,

$$
r_{c}\left(\alpha_{0}\right)=\frac{2-\sqrt{3+\alpha_{0}^{2}}}{1+\alpha_{0}}=0.182 \ldots=: r_{0} .
$$

For $\alpha_{0} \leqq \alpha<1$,

$$
r_{c}(\alpha)=\frac{2-\sqrt{3+\alpha^{2}}}{1+\alpha} .
$$

So for each function $f(z) \in T R$ in the disc $|z| \leqq r_{c}(\alpha)$ the inequality

$$
\operatorname{Re}\left(1+\frac{z f^{\prime \prime}(z)}{f^{\prime}(z)}\right) \geqq \alpha \quad 0 \leqq \alpha<1,
$$

holds with equality only for the following extremal functions:

a) in the case $0 \leqq \alpha<\alpha_{0}$ for the function (2) at the "critical points" $z= \pm i r_{c}(\alpha)$.

b) in the case $\alpha=\alpha_{0}$ for the function (2) at the "critical points" $z= \pm \operatorname{ir}_{c}(\alpha)$ and for the Koebe functions (6) at the "critical points" $z= \pm r_{c}(\alpha)$, respectively.

(c) in the case $\alpha_{0}<\alpha<1$ for the Koebe functions (6) at the "critical points" $z= \pm r_{c}(\alpha)$, respectively. 
Corollary. For $\alpha=0$ the radius of convexity $r_{c}:=r_{c}(0)$ of the class $T R$ of typically real functions (1) is

$$
r_{c}=\sqrt{6}-\sqrt{5}=0.213 \ldots
$$

Thus for each function $f(z) \in T R$ in the disc $|z| \leqq \sqrt{6}-\sqrt{5}$ the inequality

$$
\operatorname{Re}\left(1+\frac{z f^{\prime \prime}(z)}{f^{\prime}(z)}\right) \geqq 0
$$

holds with equality only for the function (2) at the "critical points" $z= \pm i(\sqrt{6}-\sqrt{5})$.

Proof. I. According to the same Ruscheweyh theorem ([2], Theorem 1a, pp. $19-20)$, the minimum of the functional $\operatorname{Re}\left(1+\left(z f^{\prime \prime}(z)\right) / f^{\prime}(z)\right)$ in the class $T R$ is also attained for the functions (7). From (7) we obtain

where we have set

$$
f^{\prime}(z)=\frac{\left(1-z^{2}\right)\left(1-2 \tau_{c} z+z^{2}\right)\left(1-2 \bar{\tau}_{c} z+z^{2}\right)}{\left(1-2 t_{1} z+z^{2}\right)^{2}\left(1-2 t_{2} z+z^{2}\right)^{2}}
$$

$$
\tau_{c}=\frac{t_{1}+t_{2}}{2}+\frac{t_{2}-t_{1}}{2} e^{i \varphi}, \quad \bar{\tau}_{c}=\frac{t_{1}+t_{2}}{2}+\frac{t_{2}-t_{1}}{2} e^{-i \varphi}
$$

and

$$
\mu=\cos ^{2} \frac{\varphi}{2}, \quad 0 \leqq \varphi \leqq \pi .
$$

Via the logarithmic derivative of (43) we obtain the formula

$$
1+\frac{z f^{\prime \prime}(z)}{f^{\prime}(z)}=2 \gamma\left(t_{1}, z\right)+2 \gamma\left(t_{2}, z\right)-\gamma\left(1, z^{2}\right)-\gamma\left(\tau_{c}, z\right)-\gamma\left(\bar{\tau}_{c}, z\right),
$$

with the notation (10). From (45) and (11) it follows that

$$
\operatorname{Re}\left(1+\frac{z f^{\prime \prime}(z)}{f^{\prime}(z)}\right)=2 F\left(t_{1}, z\right)+2 F\left(t_{2}, z\right)-F\left(1, z^{2}\right)-F\left(\tau_{c}, z\right)-F\left(\bar{\tau}_{c}, z\right) .
$$

For a fixed $z \neq 0,|z|<1$, let us note

where

$$
m_{c}(z)=\min _{I_{c}}\left(2 F\left(t_{1}, z\right)+2 F\left(t_{2}, z\right)-F\left(1, z^{2}\right)-F\left(\tau_{c}, z\right)-F\left(\bar{\tau}_{c}, z\right)\right)
$$

$$
I_{c}=\left\{\left(t_{1}, t_{2}, \varphi\right) \mid-1 \leqq t_{1} \leqq t_{2} \leqq 1, \quad 0 \leqq \varphi \leqq \pi\right\} .
$$

For $z=r e^{i \theta},-\pi \leqq \theta \leqq \pi$, we obtain from (10) and (11) the representation

$$
F(u, z)=\operatorname{Re} \frac{b}{a-u}
$$


where

$$
\begin{aligned}
& a:=\operatorname{ch}\left(\ln \frac{1}{z}\right)=\frac{1}{2}\left(\frac{1}{z}+z\right)=\sigma \cos \theta-i \varrho \sin \theta \\
& b:=\operatorname{sh}\left(\ln \frac{1}{z}\right)=\frac{1}{2}\left(\frac{1}{z}-z\right)=\varrho \cos \theta-i \sigma \sin \theta
\end{aligned}
$$

and $\sigma, \varrho$ are given by (14).

Above (see properties a), b) and c) in the proof of Theorem 1) we have studied the function (46) for real $u=t,-1 \leqq t \leqq 1$ and fixed $z=r e^{i \theta}$. Now we shall examine the sum

$$
H\left(u_{1}, u_{2}, z\right):=F(u, z)+F(\bar{u}, z)
$$

for the complex conjugates $u=u_{1}+i u_{2}$ and $\bar{u}=u_{1}-i u_{2}$ with $|u| \leqq 1$ and fixed $z=r e^{i \theta}, 0<r<\sqrt{2}-1,-\pi \leqq \theta \leqq \pi$. From (46) and (48) we obtain

and hence

$$
\frac{\partial^{2} H}{\partial u_{1}^{2}}=-\frac{\partial^{2} H}{\partial u_{2}^{2}}=2 \operatorname{Re} \frac{b}{(a-u)^{3}}+2 \operatorname{Re} \frac{b}{(a-\bar{u})^{3}}
$$

$$
\frac{\partial^{2} H}{\partial u_{1}^{2}}+\frac{\partial^{2} H}{\partial u_{2}^{2}}=0
$$

Thus from (49) and (50) it follows that for the considered fixed $z$ the sum (48) is a harmonic function with respect to $u_{1}$ and $u_{2}$ in the disc $|u| \leqq 1$. Therefore the maximum principle of harmonic functions, applied to $H\left(u_{1}, u_{2}, z\right)$ in the disc $|u| \leqq 1$, says that the sum (48) is maximal on the circle $|u|=1$. The last condition for the variables (44) with $0 \leqq \varphi \leqq \pi$ is satisfied only for the following three cases:

$$
\begin{aligned}
& t_{1}=-1,-1 \leqq t_{2} \leqq 1, \quad \varphi=\pi, \\
& t_{2}=1,-1 \leqq t_{1} \leqq 1, \quad \varphi=0, \\
& t_{1}=-1, t_{2}=1, \quad 0<\varphi<\pi .
\end{aligned}
$$

Now from the properties a), b) and c) in the proof of Theorem 1 and from the corresponding cases $(51-53)$ we can draw the following conclusions:

(A) If $0<r<\sqrt{2}-1$ and $0 \leqq|\theta| \leqq \psi(\sigma)$, then

$$
m_{c}(z)=2 F(-1, z)-F\left(1, z^{2}\right)
$$

with the extremal function

$$
f(z)=\frac{z}{(1+z)^{2}}
$$

From (54), (10) and (11) it follows that

$$
m_{c}\left(r e^{i \theta}\right) \geqq m_{c}(z)=\frac{2 \varrho}{\sigma+1}-\frac{\sigma}{\varrho}=\frac{\sigma-2}{\varrho}, \quad 0<r<\sqrt{2}-1,0 \leqq|\theta| \leqq \psi(\sigma),
$$

where the equality holds only for $\theta=0$. 
(B) If $0<r<\sqrt{2}-1$ and $\sigma-\psi(\sigma) \leqq|\theta| \leqq \pi$, then

$$
m_{c}(z)=2 F(1, z)-F\left(1, z^{2}\right)
$$

with the extremal function

$$
f(z)=\frac{z}{(1-z)^{2}} .
$$

From (57), (10) and (11) it follows that

$$
m_{c}\left(r e^{i \theta}\right) \geqq m_{c}(-r)=\frac{2 \varrho}{\sigma+1}-\frac{\sigma}{\varrho}=\frac{\sigma-2}{\varrho}, \quad 0<r<\sqrt{2}-1, \pi-\psi(\sigma) \leqq|\theta| \leqq \pi,
$$

where the equality holds only for $|\theta|=\pi$.

(C) If $0<r<\sqrt{2}-1$ and $\psi(\sigma)<|\theta|<\pi-\psi(\sigma)$, then

$$
\begin{aligned}
m_{c}(z) & =2 F(-1, z)+2 F(1, z)-F\left(1, z^{2}\right)-F\left(e^{i \varphi_{0}}, z\right)-F\left(e^{-i \varphi_{0}}, z\right) \\
& =3 F\left(1, z^{2}\right)-F\left(e^{i \varphi_{0}}, z\right)-F\left(e^{-i \varphi_{0}}, z\right)=: m\left(\varphi_{0}, z\right)
\end{aligned}
$$

for a certain $\varphi_{0}, 0<\varphi_{0}<\pi$ with the extremal function

$$
f(z)=\cos ^{2} \frac{\varphi_{0}}{2} \cdot \frac{z}{(1+z)^{2}}+\sin ^{2} \frac{\varphi_{0}}{2} \cdot \frac{z}{(1-z)^{2}} .
$$

From (46) we obtain

$$
F\left(e^{i \varphi_{0}}, z\right)+F\left(e^{-i \varphi_{0}}, z\right)=\operatorname{Re} \frac{2 b\left(a-t_{0}\right)}{a^{2}-2 a t_{0}+1}
$$

where

$$
t_{0}=\cos \varphi_{0}, \quad 0<\varphi_{0}<\pi .
$$

For fixed $r(0<r<\sqrt{2}-1)$ and $\theta(0<|\theta|<\pi)$ and the corresponding $a$ and $b$, determined by (47) and (14), the Moebius transformation

$$
\delta:=\frac{2 b(a-t)}{a^{2}-2 a t+1}
$$

maps the real axis of the complex $t$-plane onto the circle

$$
\left|\delta-i \frac{|b|^{2} \bar{b}-2 b\left(|a|^{2}-1\right)}{2\left(|a|^{2}-1\right) \operatorname{Im} a}\right|=\frac{|b|^{3}}{2\left(|a|^{2}-1\right)|\operatorname{Im} a|}
$$

of the complex $\delta$-plane. From (65) it follows that

$$
\operatorname{Re} \delta \leqq \frac{\left(|b|^{2}+2\left(|a|^{2}-1\right)\right)|\operatorname{Im} b|+|b|^{3}}{2\left(|a|^{2}-1\right)|\operatorname{Im} a|}
$$

Thus, from (62-66) with (47) and (14) in mind, we obtain the inequality

$$
F\left(e^{i \varphi_{0}}, r e^{i \theta}\right)+F\left(e^{-i \varphi_{0}}, r e^{i \theta}\right) \leqq \frac{\sigma\left(3 \varrho^{2}-\sin ^{2} \theta\right)|\sin \theta|+\sqrt{\left(\varrho^{2}+\sin ^{2} \theta\right)^{3}}}{2 \varrho\left(\varrho^{2}-\sin ^{2} \theta\right)|\sin \theta|}=: \Delta(\theta)
$$


for $0<\varphi_{0}<\pi, 0<r<\sqrt{2}-1$ and $0<|\theta|<\pi$. Equality is attained for $\varphi_{0}=\pi / 2$ and $|\theta|=\pi / 2$. Finally, from (67), (60) and (46) we obtain the inequality

$$
m\left(\varphi_{0}, r e^{i \theta}\right) \geqq \frac{3 \sigma \varrho}{\varrho^{2}+\sin ^{2} \theta}-\Delta(\theta)=: G(\theta)
$$

at least on the intervals $0<\varphi_{0}<\pi, 0<r<\sqrt{2}-1$ and $\psi(\sigma)<|\theta|<\pi-\psi(\sigma)$, where equality is attained for $\varphi_{0}=\pi / 2$ and $|\theta|=\pi / 2$.

For $0<\theta \leqq \pi / 2$ and fixed $r, 0<r<\sqrt{2}-1$, the function $G(\theta)$, determined by (68) and (67), has the derivative

$$
G^{\prime}(\theta)=-\frac{\varrho \cos \theta K(\theta)}{2 \sin ^{2} \theta\left(\varrho^{4}-\sin ^{4} \theta\right)^{2}},
$$

where

$$
K(\theta)=16 \sigma \sin ^{3} \theta\left(\varrho^{4}-\varrho^{2} \sin ^{2} \theta+\sin ^{4} \theta\right)-\left(\varrho^{2}-5 \sin ^{2} \theta\right) \sqrt{\left(\varrho^{2}+\sin ^{2} \theta\right)^{5}} .
$$

The derivative $K^{\prime}(\theta)$ of the function (70) is positive for $0<\theta<\pi / 2$ and $0<r<\sqrt{2}-1$, $\sigma>\sqrt{2}, \varrho>1$. Hence for $0 \leqq \theta \leqq \pi / 2$ and fixed $r(0<r<\sqrt{2}-1)$ the function (70) increases from $K(0)=-\varrho^{7}<0$ to

$$
K\left(\frac{\pi}{2}\right)=-\sigma p_{1}\left(\sigma^{2}\right)
$$

where

$$
p_{1}(x)=x^{3}-22 x^{2}+48 x-48, \quad x=\sigma^{2} .
$$

The polynomial $p_{1}(x)$ has only one real root $x_{1}$ which lies on the interval $(19,20)$. If $\sigma_{1}=\sqrt{x_{1}}$, then $p_{1}\left(\sigma^{2}\right)<0$ for $\sqrt{2}<\sigma<\sigma_{1}$ and $p_{1}\left(\sigma^{2}\right) \geqq 0$ for $\sigma \geqq \sigma_{1}$. Thus from (71-72) and (69) we conclude that for $0<\theta<\pi / 2$ the function $G(\theta)$ has a maximum if $\sqrt{2}<\sigma<\sigma_{1}$ and it increases if $\sigma \geqq \sigma_{1}$. Hence for $\psi(\sigma) \leqq \theta \leqq \pi / 2$ we have

$$
G(\theta) \geqq \min \left(G(\psi(\sigma)), G\left(\frac{\pi}{2}\right)\right), \quad \sqrt{2}<\sigma<\sigma_{1},
$$

where the equality holds only for $\theta=\psi(\sigma)$ or $\theta=\pi / 2$. For $\sigma \geqq \sigma_{1}$,

$$
G(\theta) \geqq G(\psi(\sigma)),
$$

where the equality holds only for $\theta=\psi(\sigma)$. From (68), (67), (15) and (14) we obtain

$$
G(\psi(\sigma))=\frac{\sigma\left(3 \sigma^{2}-4\right) \sqrt{\sigma^{2}+8}-\left(3 \sigma^{4}+8\right)}{8 \sigma \varrho}
$$

and

$$
G\left(\frac{\pi}{2}\right)=\frac{\varrho\left(\sigma^{2}-6\right)}{\sigma\left(\sigma^{2}-2\right)}
$$


From (75) and (76) it follows that

$$
\begin{aligned}
G(\psi(\sigma))-G\left(\frac{\pi}{2}\right) & =\frac{\sigma\left(\sigma^{2}-2\right)\left(3 \sigma^{2}-4\right) \sqrt{\sigma^{2}+8}-p_{2}\left(\sigma^{2}\right)}{8 \sigma \varrho\left(\sigma^{2}-2\right)} \\
& =-\frac{2 p_{3}\left(\sigma^{2}\right)}{\sigma \varrho\left(\sigma^{2}-2\right)\left(\sigma\left(\sigma^{2}-2\right)\left(3 \sigma^{2}-4\right) \sqrt{\sigma^{2}+8}+p_{2}\left(\sigma^{2}\right)\right)}
\end{aligned}
$$

where

$$
p_{2}(x)=3 x^{3}+2 x^{2}-48 x+32, \quad x=\sigma^{2}
$$

and

$$
p_{3}(x)=3 x^{4}-64 x^{3}+228 x^{2}-224 x+64, \quad x=\sigma^{2} .
$$

The polynomial $p_{2}(x)$ has three real roots which lie on the intervals $(-5,-4),(0,1)$ and $(3,4)$, respectively. Let $x_{2}$ denote the roots on $(3,4)$, and $\sigma_{2}=\sqrt{x_{2}}$. Then $p_{2}\left(\sigma^{2}\right) \leqq 0$ for $\sqrt{2}<\sigma \leqq \sigma_{2}$. Thus from the first equation (77) the inequality

$$
G(\psi(\sigma))>G\left(\frac{\pi}{2}\right), \quad \sqrt{2}<\sigma \leqq \sigma_{2}
$$

follows. The polynomial $p_{3}(x)$ has four real roots lying on the intervals $(1 / 2,3 / 4)$, $(3 / 4,1),(2,3)$ and $(17,18)$, respectively. Let $x_{3}$ denote the root in $(17,18)$. Then $p_{3}(x) \leqq 0$ for $x_{2}<x \leqq x_{3}$ and $p_{3}(x)>0$ for $x>x_{3}$. Therefore, if $\sigma_{3}=\sqrt{x_{3}}$, we have $p_{3}\left(\sigma^{2}\right) \leqq 0$ for $\sigma_{2}<\sigma \leqq \sigma_{3}$ and $p_{3}\left(\sigma^{2}\right)>0$ for $\sigma>\sigma_{3}$. Thus from the second equation (77) we get the inequalities

$$
G(\psi(\sigma)) \geqq G\left(\frac{\pi}{2}\right), \quad \sigma_{2}<\sigma \leqq \sigma_{3},
$$

with equality only for $\sigma=\sigma_{3}$, and

$$
G(\psi(\sigma))<G\left(\frac{\pi}{2}\right), \quad \sigma>\sigma_{3} .
$$

Finally, for $0<\varphi_{0}<\pi, 0<r<\sqrt{2}-1$ and $\psi(\sigma)<|\theta|<\pi-\psi(\sigma)$ we obtain from (60), (68), (73-76) and (78-80)

$$
m\left(\varphi_{0}, r e^{i \theta}\right) \geqq m\left(\frac{\pi}{2}, \pm i r\right)=\frac{\varrho\left(\sigma^{2}-6\right)}{\sigma\left(\sigma^{2}-2\right)}, \quad \sqrt{2}<\sigma<\sigma_{3},
$$

with equality only for $\varphi_{0}=|\theta|=\pi / 2$, and

$$
m\left(\varphi_{0}, r e^{i \theta}\right)>\frac{\sigma\left(3 \sigma^{2}-4\right) \sqrt{\sigma^{2}+8}-\left(3 \sigma^{4}+8\right)}{8 \sigma \varrho}, \quad \sigma>\sigma_{3} .
$$

II. Now we compare the "minima" in (56), (59), (81) and (82). First, for $\sigma>\sqrt{2}(\varrho>1)$ we obtain from (75)

$$
G(\psi(\sigma))-\frac{\sigma-2}{\varrho}=\frac{4(\sigma-1) p_{4}(\sigma)}{\sigma \varrho\left(\sigma\left(3 \sigma^{2}-4\right) \sqrt{\sigma^{2}+8}+3 \sigma^{4}+8(\sigma-1)^{2}\right)}
$$

where

$$
p_{4}(\sigma)=3 \sigma^{4}-6 \sigma^{3}+2 \sigma^{2}-6 \sigma+2 \text {. }
$$


The polynomial $p_{4}(\sigma)$ has only two real roots which lie on the intervals $(0,1)$ and $(2, \sqrt{6})$. Hence

$$
G(\psi(\sigma))>\frac{\sigma-2}{\varrho}
$$

at least for $\sigma \geqq \sqrt{6}$. Thus it follows from (55-56), (58-61), (75), (81-82) and (83) that on the circle $|z|=r, 0<r<\sqrt{2}-1$,

$$
m_{c}(z) \geqq \min \left(\frac{\varrho\left(\sigma^{2}-6\right)}{\sigma\left(\sigma^{2}-2\right)}, \frac{\sigma-2}{\varrho}\right), \sigma>\sqrt{2} \quad(\varrho>1) .
$$

Equality is attained only by the corresponding extremal functions (2) and (6) at the "critical points" $z= \pm i r$ and $z= \pm r$, respectively. Second, for $\sigma>\sqrt{2}, \varrho>1$ we have by (84)

where

$$
\frac{\sigma-2}{\varrho}-\frac{\varrho\left(\sigma^{2}-6\right)}{\sigma\left(\sigma^{2}-2\right)}=-\frac{p_{0}(\sigma)}{\sigma \varrho\left(\sigma^{2}-2\right)}
$$

$$
p_{0}(\sigma)=2 \sigma^{3}-5 \sigma^{2}-4 \sigma+6 \text {. }
$$

The polynomial $p_{0}(\sigma)$ has three real roots lying on the intervals $(-2,-1),(0,1)$ and $(\sqrt{6}, 3)$. Let $\sigma_{0}$ denote the root in $(\sqrt{6}, 3)$. Then $p_{0}(\sigma) \leqq 0$ for $\sqrt{2}<\sigma \leqq \sigma_{0}$ and $p_{0}(\sigma)>0$ for $\sigma>\sigma_{0}$. Therefore it follows from (84-85) and (14) that on the circle $|z|=r=\sigma-\varrho(0<r<\sqrt{2}-1)$ we have the inequalities

$$
m_{c}(z) \geqq \frac{\varrho\left(\sigma^{2}-6\right)}{\sigma\left(\sigma^{2}-2\right)}, \quad \sqrt{2}<\sigma<\sigma_{0},
$$

with equality only for the function (2) at the points $z= \pm i$,

$$
m_{c}(z) \geqq \frac{\varrho_{0}\left(\sigma_{0}^{2}-6\right)}{\sigma_{0}\left(\sigma_{0}^{2}-2\right)}=\frac{\sigma_{0}-2}{\varrho_{0}}, \quad \varrho_{0}=\sqrt{\sigma_{0}^{2}-1},
$$

with equality only for the functions (2) and (6) at the points $z= \pm i r$ and $z= \pm r$, respectively, and

$$
m_{c}(z) \geqq \frac{\sigma-2}{\varrho}, \quad \sigma>\sigma_{0},
$$

with equality only for the functions (6) at the points $z= \pm r$.

In particular, for $\sigma=\sqrt{6}(\varrho=\sqrt{5})$ we obtain from (87) the radius of convexity $r_{c}=\sqrt{6}-\sqrt{5}$ of the class $T R$, i.e. the Corollary with $(41-42)$.

In the general case we equate the "minima" (87-89) with $\alpha$, i.e. we set

$$
\frac{\varrho\left(\sigma^{2}-6\right)}{\sigma\left(\sigma^{2}-2\right)}=\alpha
$$

and

$$
\frac{\sigma-2}{\varrho}=\alpha
$$


First, we consider the case (88). From (90) and (91) we obtain

$$
\left(1-\alpha^{2}\right) \sigma^{3}-2 \sigma^{2}-2\left(3-\alpha^{2}\right) \sigma+12=0 .
$$

For $\sigma=\sigma_{0}$ the polynomial (86) vanishes. It is easy to eliminate $\sigma$ from (92) and (86). This elimination leads us to the equation (36), four roots of which are real and lying on the intervals $(-1,0),(-\sqrt{22},-\sqrt{23}),(0,1)$ and $(\sqrt{22}, \sqrt{23})$. Hence the least positive root $\alpha_{0}=0.314 \ldots$ of (36) is the value of the "minimum" (88). Finally, for $0 \leqq \alpha<1$ we obtain from (90), (91) and (14) the equation (37) (the roots of which with respect to $r^{2}$ are on the intervals $(0,1 / 2),(1 / 2,1)$ and $\left.(1,+\infty)\right)$ and the expressions (38) and (39) which yield the radii of the discs where the inequalities (40) hold with the extremal functions (2) and (6). This completes the proof of Theorem 4.

A simple proof of the Corollary. By means of our method in [3], pp. 336-338, we shall yield an alternate proof of the Corollary. Here from point $(c)$, p. 101 we continue in the following way: From (60), (46), (47) and (14) we obtain, with the notation (63),

where

$$
\begin{aligned}
P\left(t_{0}\right)= & 4 \sigma\left(2 \sigma^{2}+4 \cos ^{2} \theta-3\right) t_{0}^{2}-2 \cos \theta\left(3 \sigma^{4}+8 \sigma^{2} \cos ^{2} \theta+\cos ^{4} \theta\right) t_{0} \\
& +\sigma\left(\sigma^{4}+2\left(7 \cos ^{2} \theta-4\right) \sigma^{2}+9 \cos ^{4} \theta-16 \cos ^{2} \theta+12\right) .
\end{aligned}
$$

For $\cos \theta=0$ the polynomial (94) is non-negative if $\sigma=\sqrt{6}$. For $\sigma=\sqrt{6}$ the polynomial (94) is reduced to the polynomial

$$
\begin{gathered}
Q\left(t_{0}\right):=4 \sqrt{6}\left(4 \cos ^{2} \theta+9\right) t_{0}^{2}-2 \cos \theta\left(\cos ^{4} \theta+48 \cos ^{2} \theta+108\right) t_{0} \\
+\sqrt{6} \cos ^{2} \theta\left(9 \cos ^{2}+68\right) .
\end{gathered}
$$

The equation $Q^{\prime}\left(t_{0}\right)=0$ yields the value

$$
t_{0}^{*}=\frac{\cos \theta\left(\cos ^{4} \theta+48 \cos ^{2} \theta+108\right)}{4 \sqrt{6}\left(4 \cos ^{2} \theta+9\right)}
$$

for which (95) takes its minimum. From (95) and (96) we obtain

$$
Q\left(t_{0}^{*}\right)=-\frac{\cos ^{2} \theta}{4 \sqrt{6}\left(4 \cos ^{2} \theta+9\right)} q(\cos \theta)
$$

where

$$
q(x)=x^{8}+96 x^{6}+1656 x^{4}+1896 x^{2}-3024, \quad x=\cos x .
$$

The polynomial $q(x)$ has only one pair of opposite roots which lie on the intervals $(-1,0)$ and $(0,1)$. On the other hand, we obtain from (15) for $\sigma=\sqrt{6}$ and from (98).

$$
q(\cos \psi(\sqrt{6}))=16(8068-1787 \sqrt{21})<0 .
$$

Thus from (97-99) it follows that

$$
Q\left(t_{0}^{*}\right) \geqq 0, \quad \psi(\sqrt{6})<|\theta|<\pi-\psi(\sqrt{6}),
$$


where the equality holds only for $|\theta|=\pi / 2$, i.e. according to (96) for $t_{0}^{*}=0$. From (100) we conclude that the polynomial (95) is non-negative for these $\theta$, i.e.

$$
Q\left(t_{0}\right) \geqq 0, \quad \psi(\sqrt{6})<|\theta|<\pi-\psi(\sqrt{6}),
$$

where the equality holds only for $t_{0}=0$ and $|\theta|=\pi / 2$. Finally (101), (93), (63), (61) and (14) yield

$$
m_{c}(z) \geqq 0, \quad \sigma=\sqrt{6}, \quad-1<t_{0}<1, \quad \psi(\sqrt{6})<|\theta|<\pi-\psi(\sqrt{6}),
$$

where the equality is attained only by the function (2) at the points $z= \pm i(\sqrt{6}-\sqrt{5})$. Now we compare (102) with (56) and (59) for $\sigma=\sqrt{6}$ and obtain

$$
m_{c}\left(r e^{i \theta}\right) \geqq m_{c}( \pm i r)=0, \quad r=\sqrt{6}-\sqrt{5},-\pi<\theta \leqq \pi .
$$

Equality is attained only by the function (2) at the points $z= \pm i(\sqrt{6}-\sqrt{5})$. Hence for each function $f \in T R$ on the circle $|z|=\sqrt{6}-\sqrt{5}$ the inequality (42) holds, with equality only for the function (2) at the points $z= \pm i(\sqrt{6}-\sqrt{5})$. Since the left-hand side of (42) is a harmonic function in the closed disc $|z| \leqq \sqrt{6}-\sqrt{5}$, then according to the minimum principle for harmonic functions, the sharp inequality (42) holds (without the equality sign) in the open disc $|z|<\sqrt{6}-\sqrt{5}$. Hence the radius of convexity of the class $T R$ is the number (41). This completes the second proof of the Corollary.

Analogously to Theorems 2 and 3 we can obtain from Theorem 4 sharp inequalities for the expressions

$$
\operatorname{Re}\left(1+\frac{z f^{\prime \prime}(z)}{f^{\prime}(z)}\right)
$$

and

$$
\left|\frac{f^{\prime}\left(r e^{i \theta}\right)}{f^{\prime}\left(\varrho e^{i \theta}\right)}\right|
$$

\section{References}

[1] Kinwan, W. E.: Extremal problems for the typically real functions. - Amer. J. Math. 88, 1966, 942-954.

[2] Ruscheweyh, St.: Nichtlineare Extremalprobleme für holomorphe Stieltjesintegrale. - Math. Z. $142,1975,19-23$.

[3] Todorov, P. G.: A simple proof of the Kirwan theorem for the radius of starlikeness of the typically real functions and one new result. - Acad. Roy. Belg. Bull. Cl. Sci. (5), Tome LXVI, 1980-4, 334-342.

[4] Todorov, P. G.: The radii of starlikeness and convexity of order alpha of the typically real functions. - Abstracts Amer. Math. Soc. 2, 1981, 297 (81T-30-148).

Paissii Hilendarski University

Department of Mathematics

4000 Plovdiv

Bulgaria

Received 23 June 1982 